\title{
A New Algorithm for Human Face Detection Using Skin Color Tone
}

\author{
Hewa Majeed Zangana ${ }^{1}$, Imad Fakhri Al-Shaikhli ${ }^{2}$ \\ ${ }^{I}$ (Department of Information System, KICT/ International Islamic University Malaysia, Malaysia) \\ ${ }^{2}$ (Department of Computer Science, KICT/ International Islamic University Malaysia, Malaysia)
}

\begin{abstract}
Human face recognition systems have gained a considerable attention during last decade due to its vast applications in the field of computer and advantages over previous biometric methods. There are many applications with respect to security, sensitivity and secrecy. Face detection is the most important and first step of recognition system.

This paper introduces a new approach to face detection systems using the skin color of a subject. This system can detect a face regardless of the background of the picture, which is an important phase for face identification. The images used in this system are color images which give additional information about the images than the gray images provide.

In face detection, the two respective classes are the "face area" and the "non-face area". This new approach to face detection is based on color tone values specially defined for skin area detection within the image frame.

This system first resizes the image, and then separates it into its component $R, G$, and $B$ bands. These bands are transformed into another color space which is YCbCr space and then into YC'bC'r space (the skin color tone). The morphological process is implemented on the presented image to make it more accurate. At last, the projection face area is taken by this system to determine the face area.

Experimental results show that the proposed algorithm is good enough to localize a human face in an image with an accuracy of $92.69 \%$.
\end{abstract}

Keywords - Color Space, Face detection, Skin Color.

\section{Introduction}

When you are talking to a person, you usually look at his face; the expression of a person's face plays a very important role when communicating with other people. Due to its uniqueness, the face is also the most important and effective characteristics for recognizing a person.

Compared with fingerprints or retinas, taking a face image from a person is very easy. Therefore face recognition has become one of the most popular applications in the field of computer vision [1]. With the recent major terrorist attacks in the civil world, there have been increasingly substantial interests in the development of intelligent surveillance cameras that can automatically detect and recognize known criminals as well as suspicious characters. Due to such uncertain times, humans are beginning to seek support from computer systems to aid in the process of identification and location of faces in everyday scenes [2].

Images of faces vary considerably depending on lightning, occlusion, pose, facial expression, and identity. Color transforms must be implemented to deal with all remaining variation in distinguishing face skin color.

In this paper a face detection system is proposed and implemented using a skin region analysis method, within the image according to $\mathrm{YCbCr}$ color, where in each pixel is precisely classified as being either skin or non-skin. This system will detect the face from any background. This system is implemented using the scientific language which is efficient to deal with matrices mathematic operation and image processing.

For detecting face there are various algorithms including skin color based algorithms. Color is an important feature of human faces. Using skin-color as a feature for tracking a face has several advantages. Color processing is much faster than processing other facial features. Under certain lighting conditions, color is orientation invariant. This property makes motion estimation much easier because only a translation model is needed for motion estimation. However, color is not a physical phenomenon; it is a perceptual phenomenon that is related to the spectral characteristics of electromagnetic radiation in the visible wavelengths striking the retina. Tracking human faces using color as a feature has several problems like the color representation of a face obtained by a camera which is influenced by many factors (ambient light, object movement, etc.), different cameras produce significantly different color values even for the same person under the same lighting conditions and skin color differs from person to person. In order to use color as a feature for face tracking, we have to solve these problems. It is also robust towards changes in orientation and scaling and can tolerate occlusion well. A disadvantage of the color cue is its sensitivity to illumination color changes and, especially in the case of RGB, 
sensitivity to illumination intensity. One way to increase tolerance towards intensity changes in images is to transform the RGB image into a color space whose intensity and chromaticity are separate and use only chromaticity part for detection.

In this paper we have presented a comparative study of three well known skin color face localization/detection algorithms and have produced a new algorithm based on skin color classification in $\mathrm{YCbCr}$ color model. Our results show that we can localize the face more effectively by using the proposed algorithm.

\section{Color Models}

The aim of color model is to facilitate the specification of color in a certain standard. In general color model is a specification of a coordinate system; this sub-space color is used to represent each color in a single point.

\subsection{RGB Color Space}

RGB is based on the Cartesian coordinate system which is an aid cube as shown in Figure 1. The cube has the RGB values in the three corners - colors like cyan, magenta and yellow and the other three corners - the black and white at the origin is at the corner farthest from the origin. The gray scale is located on the line joining black and white. They are called additive "primary" because the colors are summed to produce the desired color. Due to the high correlation between color components: red, green and blue, as each component is subject to the effect of luminance of the light intensity of the environment, so that suffers dissatisfaction on the part of many image processing applications. In practical, this model is not well suited to describe colors in terms of human interpretation [3].

\subsubsection{Algorithm of Skin Color Based Face Detection in RGB Color Space}

Skin color is the most important feature of the face and is unique because of its color ingredients. Skin color pixels can be easily detected using standard color histogram which is normalized for every future change in the intensity of luminance of the division. And thus RGB vector is transformed into a vector [r, g] color standard which in turn provides a rapid means of detecting the skin. This gives the skin color region, which localizes face. As indicated early RGB suffers from the effect of luminance but is still able to allow us to eliminate some colors which are clearly outside the scope of normal skin color. After review and analysis of different levels of the RGB color space, it was found that the following rule works well in eliminating some redundant pixels that are labeled as non-face [4].

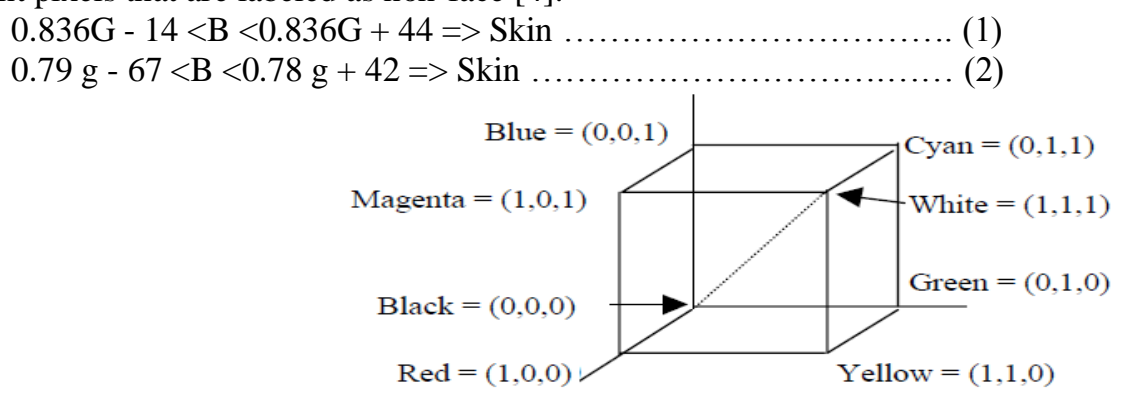

Fig. 1: RGB Color Cube

\subsection{YCbCr Color Space}

This color space was defined to meet the growing demand of digital processing algorithms of video information and has become widely used color space in digital videos. It has three components, two of them are the chrominance and luminance is one. This model comes in the family of television transmission color space along with YUC and YIQ and is designed for space analogue PAL and NTSC [4].

$\mathrm{YCbCr}$ color model has been developed to allow the transmission of color information on televisions keeping in mind that the existing television in black and white still displays images in shades of gray, has the characteristic of luminance and isolate color information, and is used in many applications such as compression.

\subsubsection{Algorithm of Skin Color Based Face Detection in $\mathrm{YCbCr}$ Color Space}

In this model, the skin color pixels belonging to the region exhibit similar $\mathrm{Cb}$ and $\mathrm{Cr}$ values as it is the skin color model based on the $\mathrm{Cr}$ and $\mathrm{Cb}$ values which can provide good coverage of human races. $\mathrm{YCbCr}$ signals that are created from the corresponding gamma adjusted RGB source using two defined constants KB and KR. 
After gamma correction and before scaling and offsets YCbCr signals are called Y1, PB, and PR and are defined as indicated below.

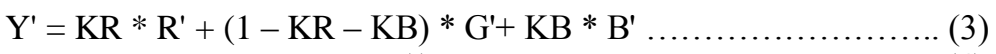

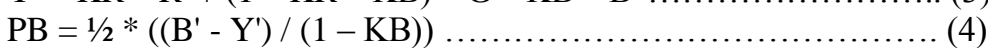

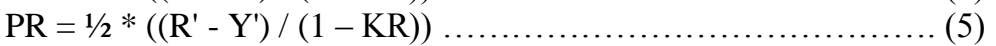

Here, the prime ' symbols mean gamma correction is being used, thus R', G' and B' nominally range from 0 to 1 , with 0 representing the minimum intensity (e.g., for display of the color black) and 1 the maximum (e.g., for display of the color white). From this the resulting luminance signal (Y) value will then have a nominal range from 0 to 1 . And the chrominance value (PB and $\mathrm{PB}$ ) values will then have a nominal range from -0.5 to +0.5 .

For representing signals in digital form, rounding off and scaling and offsets are added. For instance scaling and offset applied to the $\mathrm{Y}^{\prime}$ component per specification results in the value of 16 for black and the value of 235 for white when using an 8-bit representation. The standard has 8-bit digitized versions of CB and CR scaled to a different range of 16 to 240 . As a result, rescaling by the fraction $(235-16) /(240-16)=219 / 224$ is mandatory when doing color matrixing or processing in $\mathrm{YCbCr}$ space, leading quantization distortions when the successive processing is not executed using higher bit depths.

The form of $\mathrm{Y}^{\prime} \mathrm{CbCr}$ that was defined for standard-definition television use in the ITU-R BT.601 (formerly CCIR 601) standard for use with digital component video is derived from the corresponding RGB space as follows:

$\mathrm{KB}=0.114$

$\mathrm{KR}=0.299$

From the above constants and formulas, the following can be derived for ITU-R BT.601. Analog $\mathrm{YPbPr}$ from analogue $\mathrm{R}^{\prime} \mathrm{G}^{\prime} \mathrm{B}^{\prime}$ is derived as follows:

$$
\begin{aligned}
& \mathrm{Y}^{\prime}=0.299 * \mathrm{R}^{\prime}+0.587 * \mathrm{G}^{\prime}+0.114 * \mathrm{~B}^{\prime} \\
& \mathrm{PB}=-0.168739 * \mathrm{R}^{\prime}-0.331264 * \mathrm{G}^{\prime}+0.5 * \mathrm{~B}^{\prime} \\
& \mathrm{PR}=0.5 * \mathrm{R}^{\prime}-0.418688 * \mathrm{G}^{\prime}-0.81312 * \mathrm{~B}^{\prime}
\end{aligned}
$$

Note that the resultant signals range from 16 to 235 ; the values from 0 to 15 are called foot room, while the values from 236 to 255 are called head room.

Alternatively, digital $\mathrm{Y}^{\prime} \mathrm{CbCr}$ is derived from digital $\mathrm{Rd} \mathrm{Gd} \mathrm{Bd}$ ( 8 bits per sample) according to the following equations:

$$
\begin{aligned}
& \mathrm{Y}^{\prime}=16+(65.481 * \mathrm{Rd}+128.553 * \mathrm{Gd}+24.966 * \mathrm{Bd}) / 256 \\
& \mathrm{~PB}=128+(-37.797 * \mathrm{Rd}-74.203 * \mathrm{Gd}+112.0 * \mathrm{~B} \mathrm{~d}) / 256 \\
& \mathrm{PR}=128+(112.0 * \mathrm{Rd}-93.786 * \mathrm{Gd}-18.214 * \mathrm{Bd}) / 256 . \\
& \mathrm{Y}^{\prime}=16+(0.255 * \mathrm{Rd}+0.5021 * \mathrm{Gd}+0.0975 * \mathrm{Bd}) \ldots \ldots \ldots \ldots \\
& \mathrm{PB}=128+(-0.148 * \mathrm{Rd}-0.368 * \mathrm{Gd}+0.439 * \mathrm{~B} \mathrm{~d}) \ldots \ldots \ldots \\
& \mathrm{PR}=128+(0.439 * \mathrm{Rd}-0.368 * \mathrm{Gd}-0.071 * \mathrm{Bd}) \ldots \ldots \ldots \ldots
\end{aligned}
$$

The above equations are used in the coding part of the proposed algorithm. $\mathrm{Cr}$ and $\mathrm{Cb}$ are used instead of PB and PR. After studying and experimenting with various thresholds, it was finally found that the best results were found by using the following decree and otherwise assume that it is NOT skin and may be removed from further consideration [5].

$$
102<\mathrm{Cb}<128=>\text { Skin }
$$

\subsection{HSI Color Space}

This color model is ideal for hardware implementation. Human practice interpretation is described in terms of Hue Saturation and Intensity (Brightness). HSI model separates the intensity component of the color information-bearing and is an ideal tool for development processing algorithm based on the image description of the colors that are natural and intuitive to human health. HSI is based on the cylindrical coordinate, hue $(\mathrm{H})$ is shown with a 0 angle, varying from 0 to 360, the saturation (S) corresponds to a radius ranging from 0 to 1 and finally the intensity (I) varies along the $\mathrm{Z}$-axis with 0 being black and white is 1 . When the value of $\mathrm{S}$ is 0 , the color is a gray value of intensity. When the value of $\mathrm{S}$ is 1 , color is the limit of the cone tope. More saturation, the color is white / gray / black. Changing the value of the color will change the color of red that is to 00 to green which is 1200,2400 and blue to red black in 3600. And when $\mathrm{I}=0$, the color is black and therefore $\mathrm{H}$ is undefined. By varying the value of I, a color can be is darker or lighter. Shaded color comes out when the value of $\mathrm{I}$ is adjusted while $\mathrm{S}=1$ hold. 


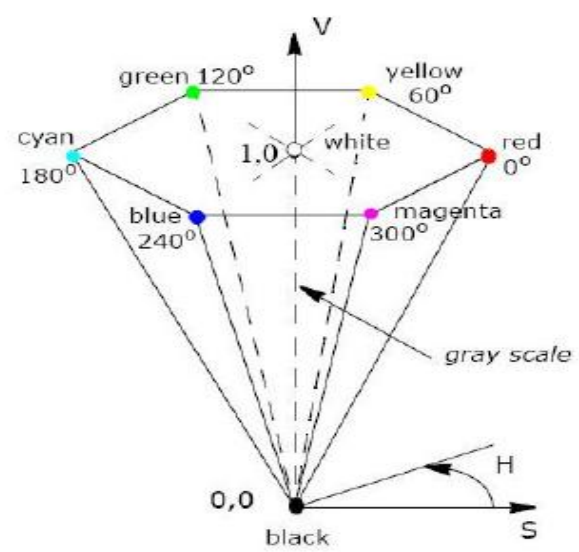

Fig. 2: Cone Model Of HSV Color Space

\subsubsection{Algorithm of Skin Color Based Face Detection in HSI Color Space}

The HSI color space is more intuitive and provides color information in the same manner that the humans think of colors and how artists mix colors in general. Dyed with saturation provides useful information regarding selective skin. Range for non-Hue face region is considered as shown below and it is also assumed to be the skin. HSI color model is represented using the following equation:

$19<\mathrm{H}<240 \Rightarrow$ Not Skin

HSI color space is defined by using the RGB as scale as transformation from RGB space to and HSI space [6]

$$
\begin{aligned}
& \mathrm{H}=360^{\circ}-\mathrm{a} / 360^{\circ} \text { if } \mathrm{b}>\mathrm{g} \\
& =\mathrm{a} / 360^{\circ} \text { otherwise } \\
& \text { Where } a=\cos ^{-1} 2 R-G-B /\left(R^{2}+G^{2}+B^{2}-R G-G B-B R\right)^{\wedge} 1 / 2 \\
& \mathrm{~S}=1-[\min (\mathrm{R}, \mathrm{G}, \mathrm{B})] / \mathrm{I} \\
& \mathrm{I}=(\mathrm{R}+\mathrm{G}+\mathrm{B}) / 3
\end{aligned}
$$

The colors are given a set of RGB color cube. The color cube is oriented so that the value component is oriented along the axis of gray, ranging from black to white. HSV are defined solely by reference to the RGB color space, they are not absolute color spaces: to specify a color precisely requires reporting not only the HSV values, but also the characteristics of the RGB space they are based, including gamma correction in use it.

Computer offers some poor cousins of these perceptual spaces which can also be found in the software interface, such as HSV. They are easy mathematical transformations RGB, and they seem to be perception systems because they use the color lightness / saturation value terminology. But take a look around, do not be fooled. Perceptual dimensions of color are poorly scaled by the color specifications that are provided in these and other systems. For example, saturation and brightness are combined, so that saturation scale may also contain a wide range of light (for example, it can evolve from white to green, which is a combination of both brightness and saturation). Similarly, the color and lightness are confounded if, for example, a saturated blue and yellow can be saturated designated as the same "lightness", but have large differences in the perceived lightness. These defects make the systems difficult to use to control the appearance of a color in a systematic approach manner. If more tweaking is needed to get the desired effect, the system offers little benefit more struggling with raw RGB specifications [7].

\section{Proposed Face Detection System}

The face detection system is presented to detect a face image from any background. This system is important in many applications such as face recognition to increase the speed and accuracy of recognition process that is because it deletes any undesired information. The proposed face detection system is depending on classifying the face as being either skin or non-skin. 


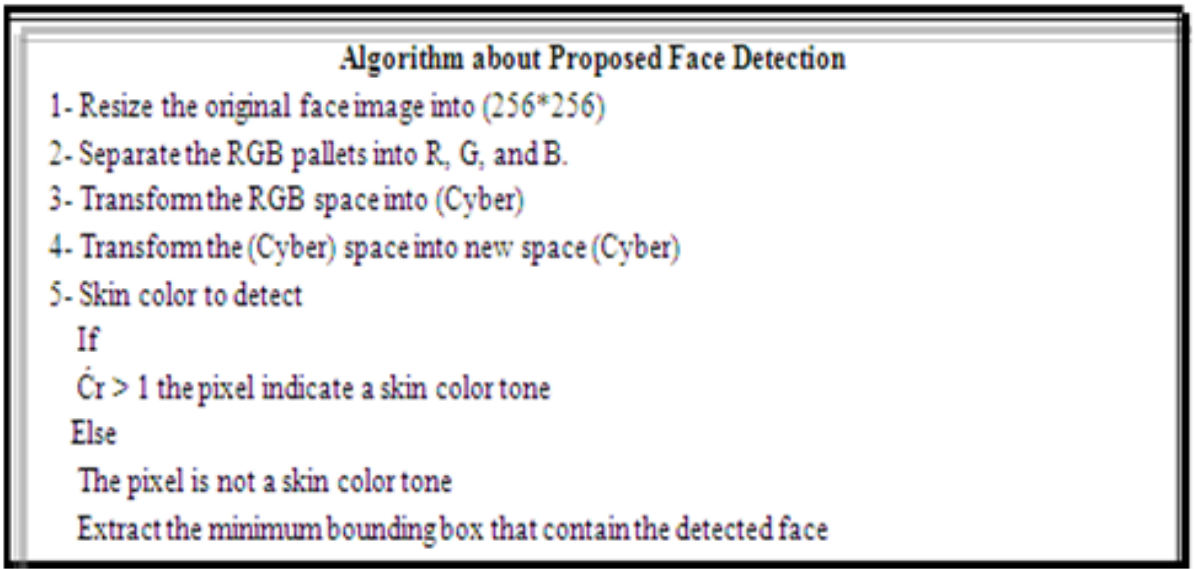

Fig. 3: Block Diagram Explaining Proposed Face Detection Algorithm

The face images are file of image entered to the system with true color (24-bit). The sizes of images are free, so we resize them into $(256 * 256)$ pixels. Then the image is decomposed into its original three color bands (Red, Green, and Blue).

Many research studies have showed that the chrominance components of the skin-tone color are independent of the luminance component [8]. However, in practice, the skin-tone color is nonlinearly dependent on luminance. The proposed detection system adopts the $\mathrm{YCbCr}$ space since it is perceptually uniform, is widely used in video compression standards (e.g., MPEG and JPEG), and it is similar to the TSL space in terms of the separation of luminance and chrominance as well as the compactness of the skin cluster. For this reason, in this stage RGB image would be converted to $\mathrm{YCbCr}$ color system using the following equation:

$$
\begin{aligned}
& \mathrm{Y}=0.299 \mathrm{R}+0.587 \mathrm{G}+0.114 \mathrm{~B} \ldots \ldots \\
& \mathrm{Cb}=(-0.169 \mathrm{R})+(-0.331 \mathrm{G})+0.5 \mathrm{~B} \\
& \mathrm{Cr}=0.5 \mathrm{R}+(-0.418 \mathrm{G})+(-0.081 \mathrm{~B})
\end{aligned}
$$

In the $\mathrm{YCbCr}$ color space, chrominance $(\mathrm{Cb}$ and $\mathrm{Cr})$ can be regarded as function of the luminance $(\mathrm{Y})$, i.e. $\mathrm{Cb}(\mathrm{Y})$ and $\mathrm{Cr}(\mathrm{Y})$. Let the new transformed chrominance be $\mathrm{C}^{\prime} b(\mathrm{Y})$ and $\operatorname{C} \operatorname{cr}(\mathrm{Y})$. The skin color model is specified by the center (denoted as $\mathrm{Cb}(\mathrm{Y})$ and $\mathrm{Cr}(\mathrm{Y})$ and spread of the cluster (denoted as $\mathrm{WCb}(\mathrm{Y})$ and $\mathrm{WCr}(\mathrm{Y}))$ as seen in following Equations, and is used for computing the transformed Chroma.

$$
\begin{aligned}
& C_{i}^{\prime}(Y)= \begin{cases}\left(C_{i}(Y)-\overline{C_{i}}(Y)\right) \frac{W_{C_{i}}}{W_{C_{i}}}+\overline{C_{i}}\left(K_{h}\right) & \text { If } Y_{1}<K_{1} \text { nr } K_{L}<Y \\
C_{i}(Y) & \end{cases} \\
& W_{C_{i}}(Y)= \begin{cases}W_{L C}+\frac{\left(Y-Y_{\min }\right)\left(W_{i}-W_{i}-Y_{\text {min }}\right)}{K_{1}} & \text { If } Y<K_{1} \\
W_{H C}+\frac{\left(Y_{\min }-Y_{i}\right)\left(W_{i}-W_{i}\right)}{Y_{\max }-K_{h}} & \text { If } K_{L}<Y\end{cases} \\
& \overline{\mathrm{C}_{\mathrm{b}}}(\mathrm{Y})= \begin{cases}108+\frac{10 \times\left(\mathrm{K}_{1}-\mathrm{Y}\right)}{\mathrm{K}_{1}-\mathrm{Y}_{\min }} & \text { If } \mathrm{Y}<\mathrm{K}_{1} \\
108-\frac{10 \times\left(\mathrm{Y}-\mathrm{K}_{\mathrm{h}}\right)}{\mathrm{Y}_{\max }-\mathrm{K}_{\mathrm{h}}} & \text { If } \mathrm{K}_{\mathrm{i}}<\mathrm{V}\end{cases} \\
& \overline{\mathrm{C}_{\mathrm{r}}}(\mathrm{Y})= \begin{cases}154-\frac{10 \times\left(\mathrm{K}_{1}-\mathrm{Y}\right)}{\mathrm{K}_{1}-\mathrm{Y}_{\min }} & \text { If } \mathrm{Y}<\mathrm{K}_{\text {I }} \\
154-\frac{22 \times\left(\mathrm{Y}-\mathrm{K}_{\mathrm{h}}\right)}{\mathrm{Y}_{\max }-\mathrm{K}_{\mathrm{h}}} & \text { If } \mathrm{K} .<\mathrm{V}\end{cases}
\end{aligned}
$$


As the color space is transformed to YC'bC'r, then the skin color tone is detected based on the C'r, when the value of C'r is larger than 1, then it indicate a skin color tone as shown in Figure 4.
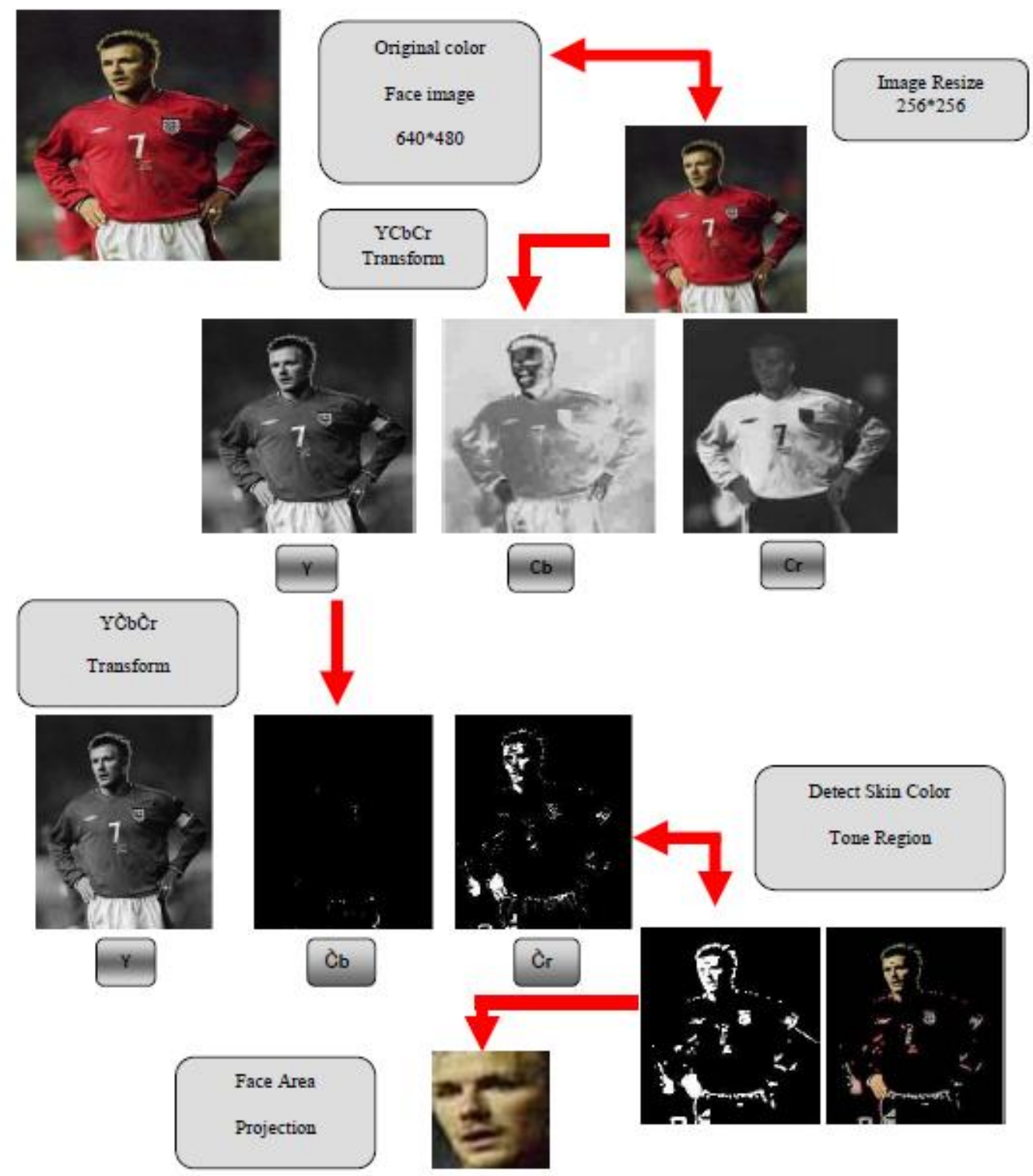

Fig. 4: The Proposed Face Detection System Stages

\section{Experimental Results}

In this section a detailed experimental comparison of the above stated algorithms has been presented using MATLAB. We used color images obtained from internet which comprise of only single face. The accuracy is obtained in all the four cases by using the following equation:

$\%$ Accuracy $=100-($ Rate of False Dismissal + Rate of False Detection $)$

\subsection{Results of Skin Color Based Face Detection in RGB Color Space}

The results of experiment carried by [9] show that RGB color space is not very much friendly with face detection based on skin color classification. The accuracy of this experiment is found to be $21.64 \%$. These results as obtained from [9] show that the false detection rate and dismissal rate are very high, thus, causing very low accuracy in detecting the face. In RGB color space it is found that it represents some non-skin region also as the skin region and hence false detection rate is quite high.

\subsection{Results of Skin Color Based Face Detection in YCbCr Color Space}

Similar experiments have been performed on the $\mathrm{YCbCr}$ color space as on RGB. The accuracy is found to be $84.61 \%$ which is far better than results from RGB color space. These results also obtained from [9] show 
that the false detection rate is high and dismissal rate is low, thus causing a bit low accuracy in detecting the face. It is found here that skin color region is more effectively extracted. This is because $\mathrm{Cb}$ and $\mathrm{Cr}$ have some distinct color range for skin region. Thus the accuracy of this algorithm is quite good.

\subsection{Results of Skin Color Based Face Detection in HSI Color Space}

Experiments show that HSI color space is also good in classifying the skin color region. The accuracy is found to be $73.8 \%$ which is nearly equivalent to results from $\mathrm{YCbCr}$ color space. These results were also obtained from [9]. Similar to $\mathrm{YCbCr}$, in this color space also, false detection rate is high and dismissal rate is low. It is found that skin color region is more effectively extracted as in $\mathrm{YCbCr}$ color space because $\mathrm{H}$ and $\mathrm{S}$ (similar to $\mathrm{Cb}$ and $\mathrm{Cr}$ ) have some distinct color range for skin region.

\subsection{Results of Proposed Algorithm}

Our experiments show very good results for proposed algorithm. The results were obtained using the previous conditional probabilities and threshold values. The false detection and false dismissal rate are shown in Table 1. The low false dismissal rate shows the robustness of the algorithm. The relative false detection is also low and this shows that it is able to distinguish between actual skin and background color with skin color appearance. The accuracy is found to be $92.69 \%$. Sample results from proposed algorithm are shown in Figure 5.

Table 1. Skin Color Classification Results for Proposed Algorithm

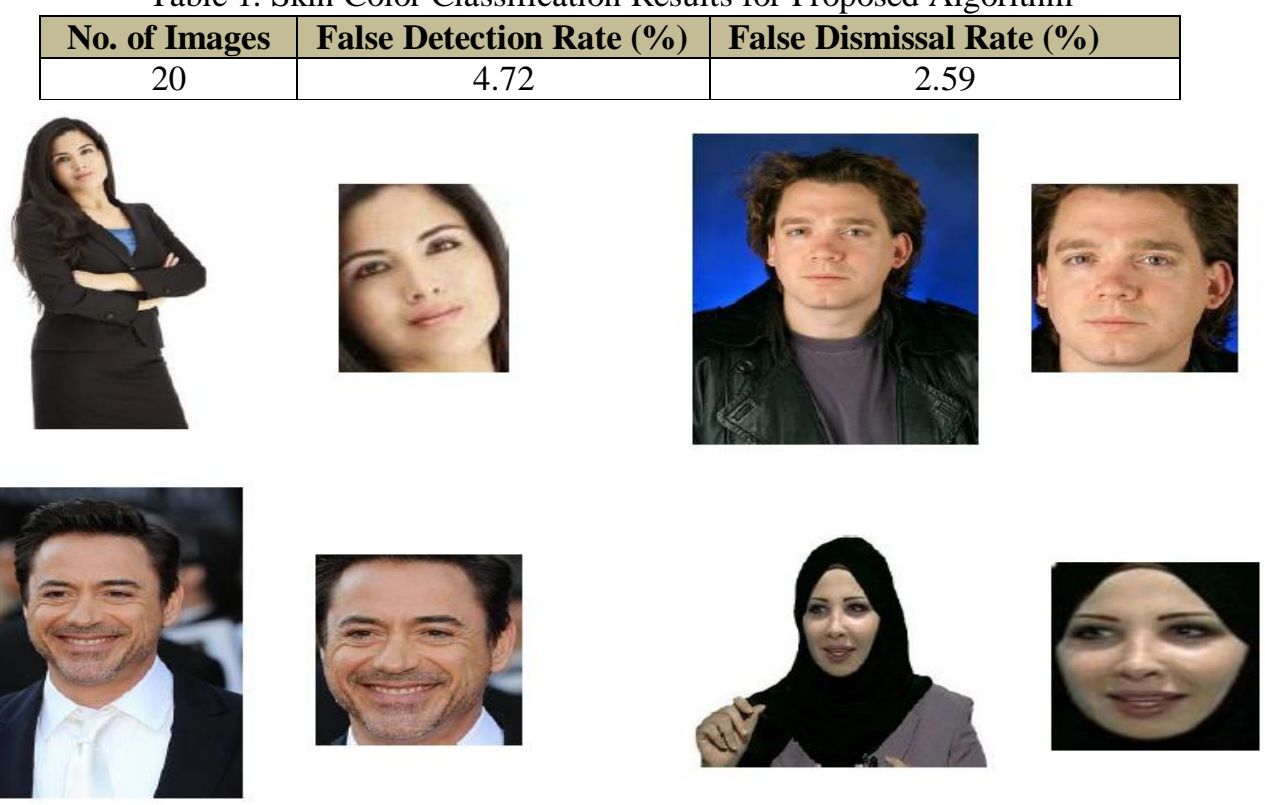

Fig. 5: Sample Results of Proposed Face Detection Algorithm

\section{Comparison Of Algorithms}

In this section comparison of the four algorithms is given. Table 2 shows the comparison of the algorithms. This comparison shows that the proposed algorithm gives good results.

Table 2. Comparison of Algorithms

\begin{tabular}{|c|c|c|c|c|}
\hline Criterion & $\begin{array}{c}\text { RGB Color Space } \\
\text { [9] }\end{array}$ & $\begin{array}{c}\text { YCbCr Color Space } \\
\text { [9] }\end{array}$ & $\begin{array}{c}\text { HSI Color Space } \\
\text { [9] }\end{array}$ & $\begin{array}{c}\text { Proposed } \\
\text { Algorithm }\end{array}$ \\
\hline Accuracy & $21.64 \%$ & $84.61 \%$ & $73.8 \%$ & $92.69 \%$ \\
\hline
\end{tabular}

\section{Conclusions}

From the face detection system proposed in this paper we got the following conclusions:

1. A face detection algorithm for color images has been proposed using a skin-tone color model and facial texture features. It overcomes the difficulty of detecting the low-luma and high-luma skin tones by applying a nonlinear transform to the $\mathrm{YCbCr}$ color space. Proposed method detects skin regions over the entire image, and then generates face candidates based on the spatial arrangement of these skin patches, our goal 
is to design a system that detects faces and facial features, allows users to edit detected faces, and use these detected facial features as indices for identification and retrieval from image databases.

2. Face detection is accomplished by first performing a skin detection search of the input image based on color segmentation. Although skin colors differ from person to person, and race to race, it was found that the color remains distributed over a very small region in the chrominance plane.

3. It deals with color scale face images instead of the gray information. The gray images are avoided for two reasons: first humans easily locate faces in color images depending on skin color tone. A more pragmatic reason is that color would increase additional data source to differentiate between person to person and between the same persons in different light sources.

4. This work has demonstrated that face image preprocessing image resize is indeed a practical solution to speed up the image searching by carefully selecting/generating proper representation features. This is believed to be the first piece of work on face image preprocessing.

5. The three different color spaces RGB, HSI and YCbCr are briefly discussed. Moreover, the objectives of this paper have been accomplished as is evident from the results which show a higher accuracy of $92.69 \%$ as compared to the accuracy of all existing algorithms.

\section{References}

[1] Hong, S. and al, e, "Facial feature detection using Geometrical face model: An efficient approach," journal of pattern recognition, Vols. 31, No. 3, pp. 273-282, 1998.

[2] Leung, C, "Real Time Face Recognition," B. Sc. Project, School of Information Technology and Electrical Engineering. University of Queesland, 2001.

[3] Sanjay Kr, Singh, D. S. Chauhan, Mayank Vatsa and Richa Singh, "A Robust Skin Color Based Face Detection Algorithm," Tamkang Journal of Science and Engineering, vol. 6 (4), pp. 227-234, 2003.

[4] Muhammad Tariq Mahmood, "Face Detection by Image Discriminating," $2006 . \quad$ [Online]. Available: http://www.bth.se/fou/cuppsats.nsf/all/6c509ae86a297ca4c12571d300512cac/\$file/DVD009-MasterThesisReport.pdf..

[5] Michael Padilla and Zihong Fan, "Automatic Face Detection Using Color Based Segmentation and Template / Energy Thresholding," 2003. [Online]. Available: http://www.stanford.edu/class/ee368/Project 03/Project/reports/ee368group16.pdf.

[6] Rafael C. Gonzalez and Richard E. Woods, Digital Image Processing. 2nded, New Jersey: Prentice-Hall, 2001 , pp. 75-103.

[7] C. A. Brewer, Color Use Guidelines for Data Representation, Alexandria: American Statistical Association, 1999 , pp. P55 -56.

[8] Sangwine, S. J. and Horne, R. E. N, The Color Image Processing Handbook, Chapman \& Hall, 1st Edition, 1998

[9] Raghuvanshi, D. S. and Agrawal, D., "Human Face Detection by using Skin Color Segmentation, Face Features and Regions Properties," International Journal of Computer Applications, vol. 38- No.9, 2012.

[10] H. H. Khaung Tin, "Robust Algorithm for Face Detection in Color Images," I.J.Modern Education and Computer Science, pp. 31-37, 2012.

[11] Tripathi, S., Sharma V. and Sharma S., "Face Detection using Combined Skin Color Detector," International Journal of Computer Applications (0975 - 8887), vol. 26- No.7, 2011.

[12] Wagner and Andrew et al, "Toward a practical face recognition system: Robust alignment and illumination by sparse representation," Pattern Analysis and Machine Intelligence, IEEE Transactions on 34.2, pp. 372-386, 2012. 